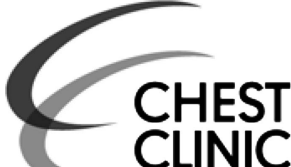

${ }^{1}$ Department of Pulmonary Medicine, Institute of Liver \& Biliary Sciences, New Delhi, India

${ }^{2}$ Department of Nephrology, Institute of Liver \& Biliary Sciences, New Delhi, India ${ }^{3}$ Department of Radiodiagnosis, Institute of Liver \& Biliary Sciences, New Delhi, India

\section{Correspondence to}

Dr Sachin Kumar, Department of Pulmonary Medicine, Institute of Liver \& Biliary Sciences, New Delhi, India; sachin.drk@gmail.com

Received 29 May 2013 Revised 9 July 2013 Accepted 21 July 2013 Published Online First 6 August 2013

IMAGES IN THORAX

\title{
Pleuropericardial effusion: a diagnostic conundrum
}

\author{
Sachin Kumar, ${ }^{1}$ Suman Lata, ${ }^{2}$ Yashwant Patidar ${ }^{3}$
}

Pleuropericardial effusion as a presenting feature raises the possibility of infectious, neoplastic and connective tissue disorders. ${ }^{1}$ However, unusual pathophysiological mechanisms may sometimes pose a diagnostic conundrum in certain clinical contexts. Simultaneous pleuropericardial effusion secondary to obstructive uropathy is such a rare scenario with no report mentioned in the published literature.

A 62-year-old man presented with progressive dyspnoea and left flank pain for 1 month. A CT scan revealed massive left-sided effusion, mild pericardial effusion (figure 1A,B) and a large staghorn calculi in the left kidney causing grade 4 hydronephrosis (figure 2A,B). Therapeutic thoracentesis revealed a straw-coloured transudative effusion with sugar of $44 \mathrm{mg} / \mathrm{dL}$, lactate dehydrogenase of $250 \mathrm{U} / \mathrm{L}$ and pleural fluid creatinine of $12.2 \mathrm{mg} / \mathrm{dL}$ against a serum creatinine of $1.5 \mathrm{mg} / \mathrm{dL}$ (ratio $8.13: 1$ ) confirming the diagnosis of urinothorax. ${ }^{2}$ His thyroid profile, autoimmune markers, HIV serology and other workup for secondary causes of pleuropericardial effusion were unrewarding. He underwent percutaneous nephrostomy followed by left nephrectomy with complete resolution of pleural and pericardial effusion and the pleural draining catheter was removed uneventfully. The common embryologic development of the pleuropericardial cavities and the existing lymphatic communications of the pleura, pericardium and diaphragm might be the pathogenetic mechanism responsible for the formation of secondary pericardial effusion in our case. ${ }^{3}$ The complete resolution of the pericardial effusion without the need for drainage after the urology procedure further establishes its direct relationship with the obstructive uropathy. Pleuropericardial effusion secondary to obstructive uropathy, though rare, thus should be kept in mind by the astute physician in the appropriate clinical context.

\section{Competing interests None.}

Provenance and peer review Not commissioned; externally peer reviewed.

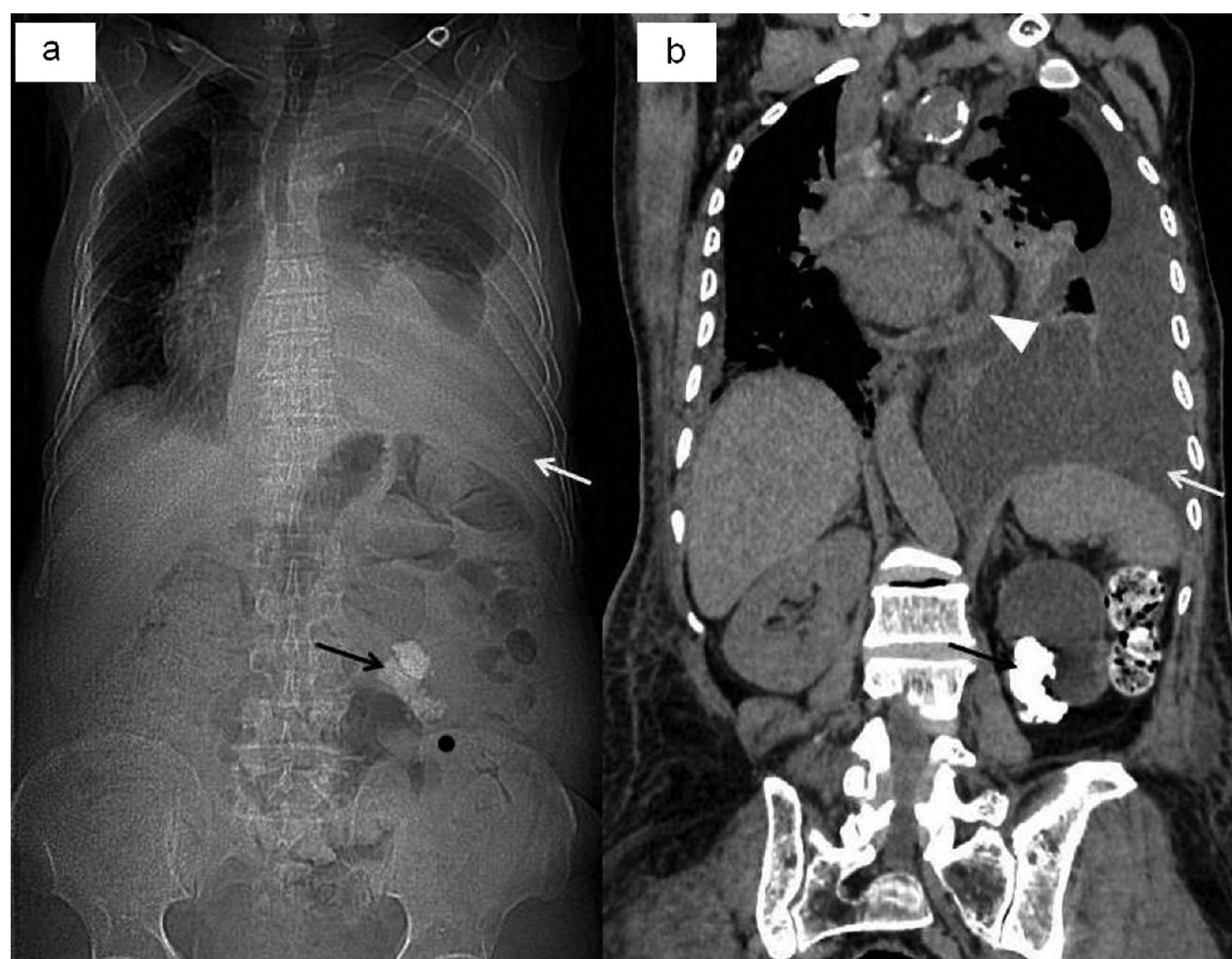

Figure 1 Scanogram (A) and oblique coronal non-contrast CT scan images of the chest and abdomen (B) showing pericardial and left pleural effusion with staghorn calculi in the left kidney (arrowheads, pericardial effusion; white arrow, pleural effusion; black arrow, staghorn calculi). 


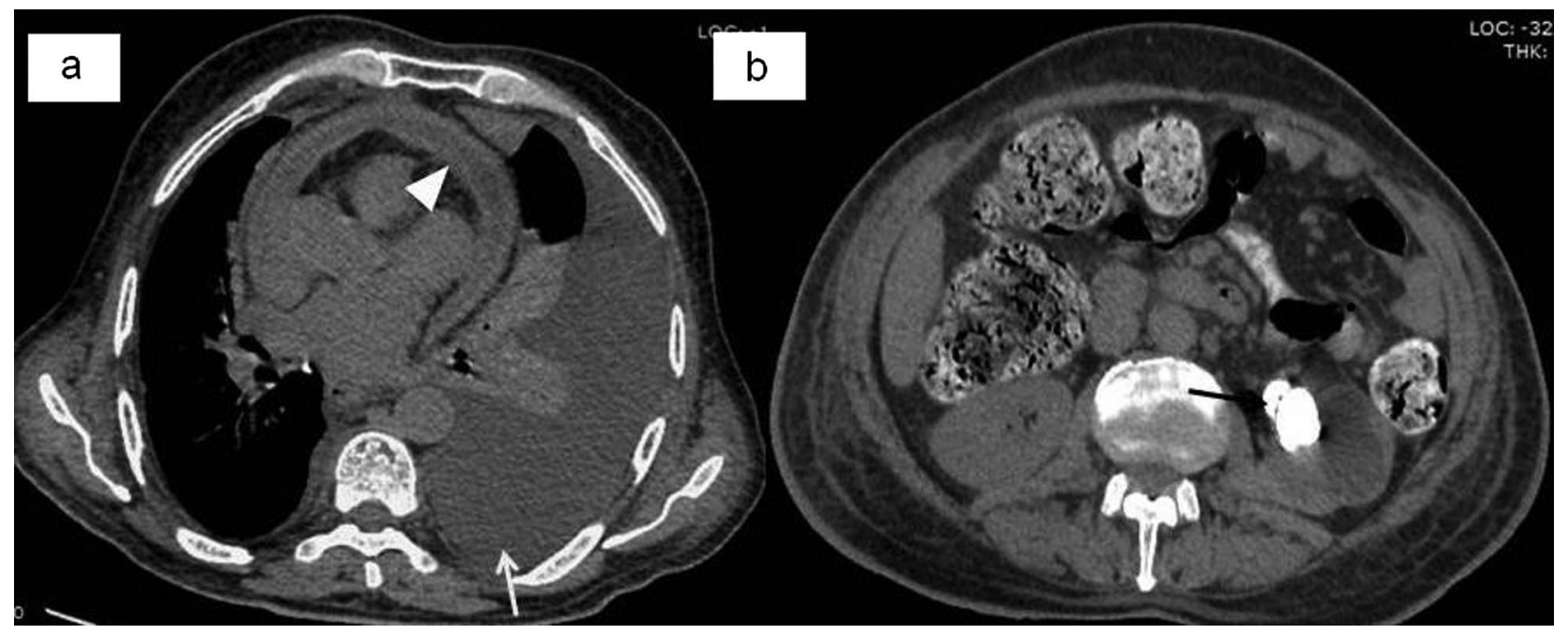

Figure 2 Axial non-contrast CT scan images of the chest (A) and abdomen (B) revealed pericardial and left pleural effusion with staghorn calculi in the left kidney causing grade 4 hydronephrosis (arrowheads, pericardial effusion; white arrow, pleural effusion; black arrow, staghorn calculi).

\section{REFERENCES}

1 Pasteur M, Laroche C, Keogan M. Pleuropericardial effusion in a 50 year old woman. Pleuropericardial effusion caused by adult inset Still's disease. Postgrad Med J 2001;77:346, 55-7
2 Garcia-Pachon E, Padilla-Navas I. Urinothorax: case report and review of the literature with emphasis on biochemical diagnosis. Respiration 2004;71:533-6.

3 Gibson AT, Segal MB. A study of the routes by which protein passes from the pericardial cavity to the blood in rabbits. J Physiol 1978;280:423-33. 